\title{
Osteocytes and Skeletal Pathophysiology
}

\author{
Jesus Delgado-Calle ${ }^{1,3} \cdot$ Teresita Bellido $^{1,2,3}$
}

Published online: 6 October 2015

(C) Springer International Publishing AG 2015

\begin{abstract}
For many years, osteocytes have been the forgotten bone cells and considered as inactive spectators buried in the bone matrix. We now know that osteocytes detect and respond to mechanical and hormonal stimuli to coordinate bone resorption and bone formation. Osteocytes are currently considered a major source of molecules that regulate the activity of osteoclasts and osteoblasts, such as RANKL and sclerostin, and genetic and pharmacological manipulations of either molecule markedly affect bone homeostasis. Besides playing a role in physiological bone homeostasis, accumulating evidence supports the notion that dysregulation of osteocyte function and alteration of osteocyte lifespan underlies the pathophysiology of skeletal disorders characterized by loss bone mass and increased bone fragility, as well as the damaging effects of cancer in bone. In this review, we highlight some of these investigations and discuss novel observations that demonstrate that osteocytes, far from being passive cells
\end{abstract}

This article is part of the Topical Collection on Molecular Biology of Skeletal Development

Teresita Bellido

tbellido@iupui.edu

Jesus Delgado-Calle

jedelgad@iupui.edu

1 Department of Anatomy and Cell Biology, Indiana University School of Medicine, 635 Barnhill Drive, MS5045L, Indianapolis, IN 46202, USA

2 Department of Medicine, Division of Endocrinology, Indiana University School of Medicine, Indianapolis, IN, USA

3 Roudebush Veterans Administration Medical Center, Indianapolis, IN, USA entombed in the bone, are critical for bone function and maintenance.

Keywords Osteocyte · Osteoclast - Osteoblast . Bone remodeling $\cdot$ Wnt signaling $\cdot$ SOST

\section{Introduction}

Osteocytes are stellate-shaped cells that differentiate from osteoblasts and become entombed within the mineralized bone matrix during bone deposition. Osteocytes are considered permanent residents in bone, with a half-life of 25 years and constitute more than $95 \%$ of bone cells within the matrix or on bone surfaces [1]. Osteocyte bodies are individually encased in lacunae carved within the mineral and exhibit cytoplasmic processes that run along narrow canaliculi within the mineralized matrix forming the so-called lacunar-canalicular system [2]. Through their cytoplasmic projections, osteocytes establish intercellular communications with neighboring osteocytes through gap junctions and also reach the periosteal and endocortical surfaces of cortical bone, the lumen of blood vessels and Haversian canals, as well as the surfaces adjacent to the bone marrow on endocortical and cancellous bone surfaces $[2,3]$. This lacunar-canalicular system has the potential to allow cell-to-cell communication between osteocytes and other bone cells and also distributes osteocytesecreted molecules within the bone/bone marrow microenvironment $[4,5]$. However, the mechanisms by which osteocyte expressed molecules and secreted factors reach their cellular targets remain poorly understood. Accumulating evidence demonstrates that osteocytes detect and respond to mechanical and hormonal stimuli to coordinate the function of osteoblasts and osteoclasts [6]. The discovery that sclerostin, a potent 
inhibitor of bone formation, is primarily expressed by osteocytes and is downregulated by bone anabolic stimuli identified this molecule as one of the long sought messengers by which osteocytes affect the function of bone surface cells [7-12]. More recent evidence demonstrates that osteocytes also express cytokines that regulate osteoclast generation and participate in the recruitment and differentiation of osteoclasts precursors, thus initiating targeted bone remodeling [13•]. This review will focus on the recent findings regarding the function of osteocytes in bone homeostasis under physiological and pathophysiological conditions.

\section{Regulation of Bone Formation by Osteocytes}

Osteocytes express several molecules that negatively affect bone formation, including the dickkopf WNT signaling pathway inhibitor 1 (DKK1) [14], also expressed by osteoblasts, and SOST, which is primarily expressed postnatally in osteocytes, but not in osteoblasts [12]. Both the product of the SOST gene sclerostin and DKK1 block the binding of the wingless-type MMTV integration site family members (Wnts) to Frizzled receptors and low-density lipoprotein receptor-related proteins (LRP) 5 and 6, thus preventing Wnt signaling activation $[12,15]$. Wnts and Wnt signaling activation have proven to be critical for osteoblast differentiation, survival, and function as they induce commitment of multipotential mesenchymal progenitors towards the osteoblast lineage, stimulate osteoblast differentiation, and regulate osteoblast activity [16•]. Thus, through the production of Wnt signaling antagonists, osteocytes are able to regulate the formation and activity of osteoblasts. Genetic and pharmacologic evidence supports this mechanism. Deletion of a single allele of the DKK1 gene in mice leads to an increase in bone formation and bone mass [17]. Similarly, targeted deletion of the SOST gene increases bone mass and bone strength [18]. In addition, genetic deletion or pharmacological inhibition of LRP4, a facilitator of sclerostin action, also results in increased bone formation and bone mass [19•]. Further, neutralizing inhibitors of DKK1, sclerostin, or LRP4 have emerged as promising and feasible therapeutic targets (discussed in section "Osteocytes, Aging and Hormonal Dysregulation").

The case of sclerostin is particularly attractive because it is also regulated by several stimuli with anabolic effects on the skeleton. For instance, parathyroid hormone (PTH), the only FDA-approved anabolic agent for osteoporosis in the USA, decreases SOST/sclerostin expression in osteocytes in rodent models and humans [11, 12, 20-22]. In addition, numerous studies support the notion that osteocytes orchestrate the increase in bone formation in response to mechanical loads by downregulating sclerostin, thereby locally unleashing Wnt signaling [23]. Cortical bone areas exposed to high mechanical strain exhibit a reduction in sclerostin-positive osteocytes and concomitant higher bone formation on adjacent periosteal surfaces [10]. Conversely, SOST/sclerostin expression levels are increased in unloaded bone [24] and mice overexpressing a human SOST transgene in osteocytes show low bone mass [7] and fail to exhibit the anabolic response to mechanical stimulation [24]. Osteocytes are considered the mechanosensors in bone and have the potential for sensing mechanical forces and translate them into biochemical signals [25]. Targeted deletion of osteocytes results in bone loss, and this osteocyte-less bone does not respond normally to unloading (discussed in section "Osteocytes and Bone Loss Induced by Reduced Mechanical Loading") [26].

Osteocytes appear also to affect osteoblast and osteoclast differentiation through physical interactions. Recent in vitro evidence suggests that direct cell-to-cell contact with osteocytes induces an upregulation of osteoblast differentiation genes (Colla, Runx2, Alpl) in osteoblasts when compared to osteoblasts cultured alone [27]. In addition, Notch signaling, which is activated by homotypic or heterotypic interactions between Notch receptors and Notch ligands, has aroused as novel pathway regulating the activity of bone cells through cell-to-cell communication [28]. Overexpression of Notch intracellular domain 1 in osteocytes decreases osteoclastmediated bone resorption and increases cancellous and cortical bone volume by mechanisms poorly understood [29-31]. Thus, cell-to-cell communication between osteocytes and other cells in bone/bone marrow microenvironment also regulates bone homeostasis, although the specific mechanisms involved are not completely understood.

New evidence demonstrates that osteocytes mediate the anabolic actions of canonical $\mathrm{Wnt} / \beta$-catenin signaling in bone [32•], demonstrating the importance of cell autonomous Wnt/ $\beta$-catenin signaling in osteocytes themselves (Fig. 1). Bone anabolic stimuli activate this pathway and human mutations of components along this pathway underscore its crucial role in bone accrual and maintenance. However, the cell responsible for orchestrating Wnt anabolic actions has remained elusive, as activation of $\mathrm{Wnt} / \beta$-catenin signaling in preosteoblasts or osteoblasts inhibits resorption without increasing bone formation [33]. This new evidence now showed that, in contrast, activation of canonical Wnt signaling in osteocytes [dominant active (da) $\beta c a t^{\mathrm{Ot}}$ mice] induces bone anabolism and triggers Notch signaling without affecting survival [32•]. These features contrast with those of mice expressing the same da $\beta$ catenin in osteoblasts, which exhibit decreased resorption and perinatal death from leukemia [16•]. da $\beta \mathrm{cat}^{\mathrm{Ot}}$ mice exhibit increased bone mineral density in the axial and appendicular skeleton and marked increase in bone volume in cancellous/ trabecular and cortical compartments compared to littermate controls. da $\beta \mathrm{cat}^{\mathrm{Ot}}$ mice display increased resorption and formation markers, high number of osteoclasts and osteoblasts in cancellous and cortical bone, increased bone matrix 


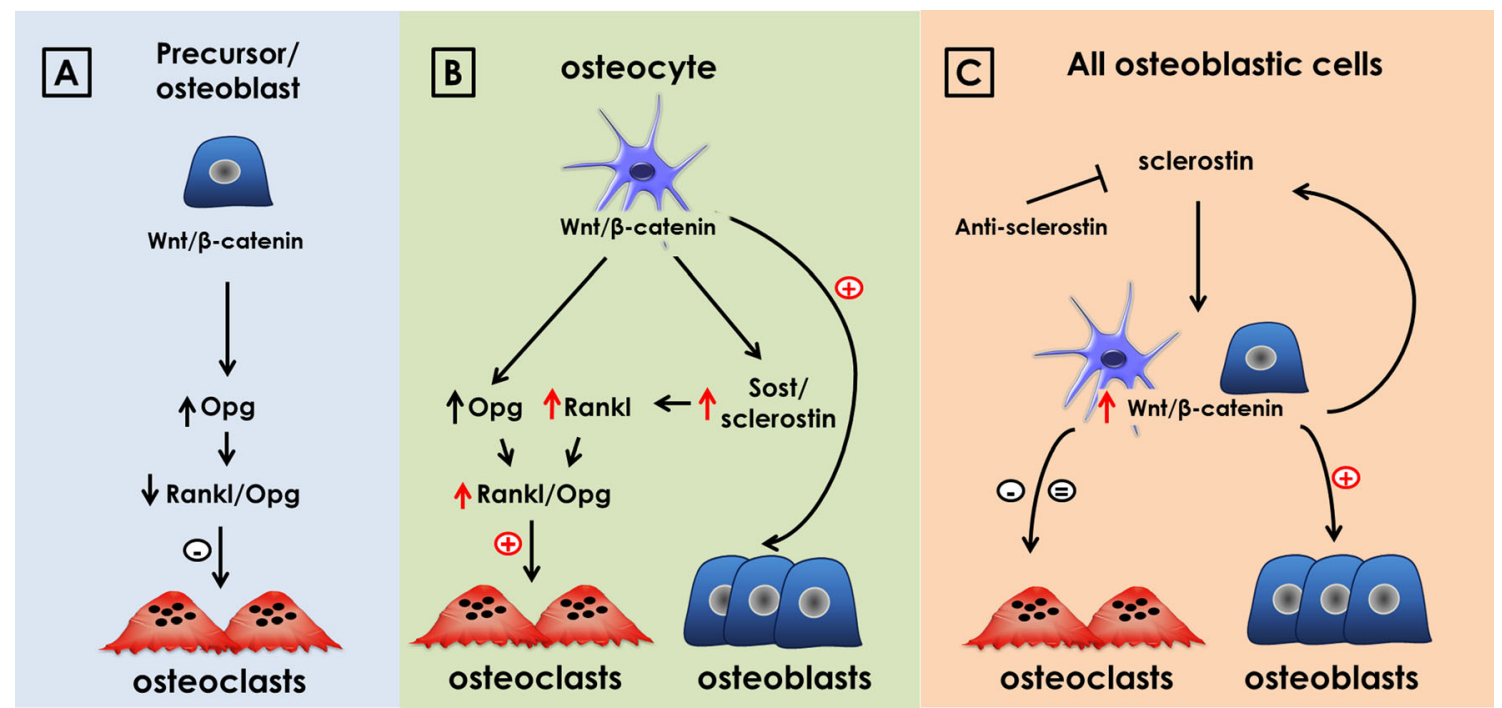

Fig. 1 Activation of Wnt- $\beta$-catenin signaling in osteocytes has an anabolic effect in bone. a Activation of Wnt/ $\beta$-catenin in osteoblasts/ osteoblast precursors increases bone mass by increasing OPG production, which reduces bone resorption, without apparent effect on osteoblast production. b Activation of $\mathrm{Wnt} / \beta$-catenin signaling in osteocytes increases both bone formation and bone resorption, with a positive balance that results in bone gain. $\mathbf{c}$ Activation of Wnt signaling in all osteoblastic cells induced by neutralizing antibodies to sclerostin increases bone formation and initially decreases the serum levels of the bone-resorption marker CTX in both mouse and clinical models that eventually come back to control levels. These findings suggest that osteocytes, rather than less differentiated cells in the osteoblastic lineage, might mediate anabolism induced by systemic activation of the $\mathrm{Wnt} / \beta$-catenin pathway, with neutralizing antibodies of sclerostin production, and markedly elevated periosteal bone formation rate. Wnt and Notch signaling target genes, osteoblast, and osteocyte markers and pro- and anti-osteoclastogenic cytokines are elevated in bones of da $\beta c a{ }^{\mathrm{Ot}}$ mice. Further, the increase in RANKL is dependent on SOST/sclerostin (discussed in section "Cross Talk Between Bone Formation and Bone Resorption via Wnt- $\beta$-catenin Signaling in Osteocytes"). Thus, activation of osteocytic $\beta$-catenin signaling increases both osteoclasts and osteoblasts leading to bone gain, and is sufficient to activate the Notch pathway. These findings demonstrate disparate outcomes of $\beta$-catenin activation in osteocytes versus osteoblasts and identify osteocytes as central target cells of the anabolic actions of canonical $\mathrm{Wnt} / \beta$-catenin signaling in bone.

Osteocytes also regulate mineralization and phosphate $(\mathrm{Pi})$ homeostasis through the release of several molecules. Osteocytes are richer than osteoblasts in molecules that regulate $\mathrm{Pi}$ homeostasis such as phosphate-regulating neutral endopeptidase (PHEX), dentin matrix protein 1 (DMP1), matrix extracellular phosphoglycoprotein (MEPE), and fibroblast growth factor 23 (FGF23), reviewed in [13•]. FGF23, mainly secreted by osteocytes, is a hormone that plays a crucial role in Pi homeostasis by inhibiting its renal reabsorption. Supraphysiologic FGF23 levels found in several genetic disorders decrease renal Pi reabsorption and induce hypophosphatemia, resulting in osteomalacia and rickets [34]. Consistent with the findings in the human diseases, transgenic mice overexpressing FGF23 are hypophosphatemic whereas FGF23 knockout mice are hyperphosphatemic $[35,36]$.

\section{Regulation of Bone Resorption by Osteocytes}

Under physiologic conditions, interactions between cells present in the bone remodeling compartment (BRC) result in a balanced and coupled remodeling of bone, a lifelong process responsible for bone damage repair and mineral homeostasis. One of the functions of the osteocyte network is to detect microdamage and trigger its repair [3]; consequently, osteocytes have a prominent role in the initiation of bone remodeling at selected sites of the skeleton. Increased osteocyte apoptosis has been shown to be induced in vivo in areas of microdamage in several pathological conditions associated with enhanced bone resorption such as overload, bone disuse, glucocorticoid administration, or estrogen deficiency, suggesting that apoptosis is linked to targeted bone resorption [37-40]. Consistent with this notion, exclusive induction of apoptosis of osteocytes is sufficient to increase resorption and leads to bone loss [26]. It has been proposed that apoptotic osteocytes trigger a cascade of signals initiated in the endosteum that contains the lining cells, which lift from the bone surface to form the canopy that encloses the BRC. Apoptotic osteocytes also signal to neighboring cells (osteocytes or other osteoblast lineage cells) to change the expression of pro- and anti-osteoclastogenic genes such as the chemotactic factor high mobility group box 1 (HMGB1) protein, which upregulates the expression of the tumor necrosis factor (ligand) superfamily, member 11 (RANKL), colonystimulating factor 1 receptor (M-CSF), or osteoprotegerin (OPG). This evidence provides the molecular basis of 
osteocyte-driven recruitment of osteoclast precursors and their differentiation in particular areas of bone [41, 42].

Osteocytes are now considered a major source in bone of the pro-osteoclastogenic cytokine RANKL, a master stimulator of osteoclastogenesis. Indeed, osteocytes express higher levels of RANKL than osteoblasts and bone marrow stromal cells, and mice lacking the RANKL gene in osteocytes exhibit an osteopetrotic phenotype characterized by reduced bone resorption $[43 \cdot, 44]$. However, it is still under debate whether the osteocytic membrane-bound or the soluble RANKL form is responsible for osteocyte-mediated bone resorption. Recent data suggest that osteocytic RANKL is predominantly provided as a membrane-bound form to osteoclast precursors through osteocyte cytoplasmatic projections, whereas the contribution of soluble RANKL to osteoclastogenesis is minor $[45,46]$. Thus, direct cell-to-cell contact between membrane-bound RANKL expressing osteocytes and osteoclast precursors appears to be required to initiate osteoclast development.

Osteocytes also secrete the anti-osteoclastogenic cytokine OPG, a soluble decoy receptor that competes with RANKL for its receptor tumor necrosis factor receptor superfamily, member 11a, NFKB activator (RANK) on osteoclast precursors [47]. OPG secretion is regulated by the $\mathrm{Wnt} / \beta$-catenin pathway, and mice lacking $\beta$-catenin in osteocytes exhibit increased osteoclast numbers and bone resorption $[48,49]$.

RANKL and M-CSF are two major factors that contribute to osteoclast differentiation and maturation. Recent evidence suggests that osteocytes are an additional source of secreted $\mathrm{M}-\mathrm{CSF}$ in bone [50]. Moreover, M-CSF expression is increased by PTH receptor signaling in osteocytes and exhibits similar pattern of expression to RANKL expression [44]. Further, deletion of the distal control region of the RANKL gene regulated by PTH (DCR) eliminates the ability of the PTH receptor/cAMP pathway to increase not only RANKL expression, as expected, but also M-CSF expression, demonstrating that M-CSF regulation is secondary to RANKL regulation [44]. This evidence is the first in vivo demonstration of MCSF regulation by RANKL and is consistent with an earlier in vitro study showing that RANKL augmented M-CSF production in pre-osteoclastic cells [51]. The regulation of MCSF by RANKL appears counter-intuitive since M-CSF increases the expression of the RANKL receptor RANK in osteoclast precursors [52], suggesting that M-CSF is needed for RANKL signaling. However, deletion of the DCR only reverses the PTH-induced M-CSF expression, demonstrating that basal levels of M-CSF are sufficient to induce RANK expression and to allow the initial action of PTH-stimulated osteocytic RANKL on bone resorption. RANKL/RANK signaling in turn could induce higher M-CSF expression in a positive feedback loop, stimulating osteoclastogenesis. Further, M-CSF is expressed not only in osteoclast precursors but also in osteocytes [53,54], suggesting that RANKL (either membrane-bound or soluble form) could upregulate M-CSF gene expression in osteoclast precursors or in osteocytes. Future studies are required to determine the cellular source of MCSF in osteocyte-driven resorption induced by PTH receptor activation.

Together, these findings suggest that osteocytes have the potential to control bone resorption through direct and indirect regulation of osteoclast differentiation and function under physiological and pathological conditions.

Besides the strong evidence demonstrating that osteocytes support osteoclast development, novel findings suggest that osteocytes, as osteoclasts, can also remove mineralized matrix by remodeling their perilacunar/canalicular matrix. The expression of genes known to be utilized by osteoclasts to remove bone, including tartrate-resistant acid phosphatase (TRAP) and cathepsin K, carbonic anhydrase 2, and matrix metalloproteinases, is elevated in osteocytes from lactating mice [55-57]. Furthermore, recent work demonstrated that global deletion of the calcitonin receptor leads to increased osteocytic, but not osteoclastic, osteolysis during lactation, providing the first evidence for a physiological role of the calcitonin receptor to protect the maternal skeleton from bone loss during lactation by direct actions on osteocytes [58]. Although the physiologic implications of these observations remain to be determined, this data suggests that, as previously described for phosphate metabolism, osteocytes also contribute to calcium mobilization and mineral homeostasis through perilacunar remodeling as has been proposed earlier[1, 59].

\section{Cross-Talk Between Bone Formation and Bone Resorption via Wnt- $\beta$-catenin Signaling in Osteocytes}

Wnt signaling pathway has emerged as a key regulator of bone homeostasis. $\beta$-catenin is the obligatory transducer for canonical Wnt signaling, which is stabilized by Wnt ligands and translocates to the nucleus to activate transcription of Wnt target genes. Inactivation of $\mathrm{Wnt} / \beta$-catenin in early stages of the osteoblastic lineage arrests osteoblast differentiation resulting in lack of mature osteoblasts and reduced bone formation [60-63]. Wnt/ $\beta$-catenin inactivation in mature osteoblasts/osteocytes increases osteoclast differentiation and bone resorption, without affecting osteoblast differentiation or bone formation [48, 49, 64]. Moreover, activation of Wnt/ $\beta$ catenin in osteoblasts increases bone mass by reducing bone resorption, without apparent effect on osteoblast production [16•]. Although this genetic evidence shows that $\mathrm{Wnt} / \beta$ catenin signaling in osteoblasts alters osteoclasts and bone resorption without effects on osteoblasts or bone formation, the pathway is linked to bone anabolism. Despite the fact that bone anabolic stimuli activate canonical Wnt/ $\beta$-catenin signaling and human mutations of this pathway underscore its 
essential role in bone accrual, the cell responsible for orchestrating these actions has remained elusive. Recent findings from our group identified osteocytes as the central target cells for the anabolic actions of canonical $\mathrm{Wnt} / \beta$-catenin signaling in bone [32•]. Activation of $\beta$-catenin signaling in osteocytes increases both bone formation and bone resorption, with a positive balance that results in bone gain. These findings suggest that osteocytes, rather than less differentiated cells in the osteoblastic lineage, might mediate anabolism induced by systemic activation of the $\mathrm{Wnt} / \beta$-catenin pathway, with inhibitors of GSK3 $\beta$ or neutralizing antibodies of sclerostin or DKK1.

We investigated the mechanism by which activation of $\beta$ catenin signaling in osteocytes versus in less mature cells of the osteoblastic lineage leads to opposite effects on resorption (Fig. 1). As expected, activation of $\mathrm{Wnt} / \beta$-catenin in both osteoblast and osteocytes increases OPG expression. However, activation of the pathway in osteocytes also increases the levels of osteocytic RANKL, resulting in a high RANKL/ OPG ratio. Upregulation of RANKL expression by activation of $\mathrm{Wnt} / \beta$-catenin in osteocytes is driven by SOST/sclerostin production, as demonstrated by the fact that SOST overexpression in vivo leads to an increase in RANKL levels [32•]. Moreover, neutralizing antibodies of sclerostin abolished this effect. This result is consistent with previous findings indicating that SOST/sclerostin upregulates RANKL and increases osteoclasts numbers [65] and supports the notion that osteocytes, through sclerostin production and $\mathrm{Wnt} / \beta$-catenin signaling modulation, not only regulate osteoblasts activity, but also control osteoclast differentiation. In fact, inhibition of sclerostin by neutralizing antibodies increases bone formation and also decreases the serum levels of the bone resorption marker CTX in both mouse and clinical models [66]. Thus, the bone anabolism achieved by administration of neutralizing antibodies of sclerostin might result from the combination of enhanced osteoblast production and reduced osteoclast numbers due to increase OPG and/or decreased RANKL production.

\section{Osteocytes and Bone Remodeling Under Pathological Conditions}

\section{Osteocytes, Aging, and Hormonal Dysregulation}

With advancing age, the balance between bone resorption and bone formation during bone remodeling changes and becomes negative. Thus, the quantity and quality of bone do not remain constant with age; rather, they change to decline bone mass and strength, leading to the common skeletal syndrome known as osteoporosis. During aging, osteocyte density declines, with an increase in the number of empty lacunae [67, $68]$. Thus, reduced osteocyte numbers and osteocyte apoptosis have been proposed to be partially responsible for the disparity between bone resorption and bone formation that occurs with aging. Reduced osteocyte density might result from the decline in physical activity with old age leading to reduced skeletal loading (discussed in section "Osteocytes and Bone Loss Induced by Reduced Mechanical Loading"), accumulation of reactive oxygen species in bone, and/or increased levels of endogenous glucocorticoids with age [3, 69, 70]. In addition, loss of sex steroids, either after loss of ovarian function in women at menopause or in patients of either sex with gonadal dysfunction, is considered the main factor for the development of osteoporosis, leading to an imbalance between bone resorption and bone formation that results in bone loss. Loss of sex steroids also increases osteocyte apoptosis $[71,72]$. Further, excess of glucocorticoids (GCs), either endogenous as in aging or due to administration as immunosuppressants, leads to loss of bone, and increased apoptosis of osteocytes (and osteoblasts) is one of the mechanisms that underlie the reduced bone formation and bone fragility that characterize GC-induced osteoporosis [73]. Therefore, decreased osteocyte viability is a common factor associated to the fragility syndrome that characterizes estrogen withdrawal, glucocorticoid excess, and aging.

Not only osteocyte viability is reduced with aging, GC excess, or loss of sex steroids, but also the activity of these cells is altered, with changes in the transcription of osteocytic genes including SOST. In fact, serum sclerostin levels increase with age, and positive associations between bone mineral density and serum sclerostin levels have been reported in several studies $[22,74]$. These results raise the possibility that increased production of sclerostin by osteocytes leads to the decreased bone formation that characterizes osteoporosis. Interestingly, however, this is not the case when SOST mRNA levels are studied in bone tissue [19•,68], suggesting that serum concentration does not necessarily reflect sclerostin levels in bone. In fact, LRP4 deficiency in bone markedly elevates serum sclerostin levels; however, animals exhibit a progressive cancellous and cortical bone gain [19•].

The fact that apoptotic osteocytes trigger bone resorption, together with the evidence suggesting that the production of osteocyte derived molecules is altered with aging, GCs, and sex steroids loss, makes osteocytes an attractive target to treat common skeletal diseases associated with bone loss (discussed in section "Osteocytes and Therapies of Common Skeletal Disorders").

\section{Osteocytes and Bone Loss Induced by Reduced Mechanical Loading}

As mentioned, mechanical forces have a positive effect on the skeleton, whereas conditions such as prolonged bed rest, physical inactivity, or reduced gravity result in bone loss and increased risk of fractures. Earlier work demonstrated that unloaded bone or bone subjected to excessive loading exhibits 
increased prevalence of osteocyte apoptosis, and it has been proposed that this event precedes RANKL upregulation and osteoclast-mediated bone resorption [37, 75-78]. Depletion of osteocytes in a murine model prevented the bone loss induced by tail suspension [26], suggesting that osteocytes are responsible for the increase in osteoclast-mediated bone resorption. Moreover, genetic deletion of RANKL in osteocytes was sufficient to protect animals from unloading-induced increased in osteoclasts and bone loss [79]. However, whether the increase in osteocyte apoptosis and RANKL expression induced by unloading, or other pro-apoptotic stimuli, are mechanistically linked had remained unknown. In a recent report, we found that in a tail suspension mouse model prevention of osteocyte apoptosis blocks the increase in RANKL expression in osteocytes, supporting the notion that indeed there is a functional relationship between osteocyte apoptosis and RANKL production [80•]. However, prevention of apoptosis in osteocytes was not sufficient to protect the animals from bone mass loss induced by unloading indicating that RANKL derived from non-osteocytic sources (likely osteoblasts) also contributes to the bone loss resulting from lack of mechanical forces. Osteocyte apoptosis is also considered a key step controlling the activation and/or targeting of osteoclastic resorption associated to bone fatigue-induced remodeling. Inhibition of osteocyte apoptosis by using a caspase inhibitor was sufficient to prevent the fatigue-induced RANKL upregulation and the activation of osteoclastic resorption [76, 81•], indicating a direct relationship between osteocyte apoptosis and local osteoclast resorption. However, these results differ from those described in our model of unloading, suggesting that the course of tissue remodeling after unloading and overloading might be determined by different mechanisms.

The levels of SOST/sclerostin are also modulated by mechanical forces. Local downregulation of SOST/sclerostin in osteocytes is required to activate $\mathrm{Wnt} / \beta$-catenin signaling and triggers bone formation in particular areas in bone subjected to mechanical forces [24]. The levels of circulating sclerostin are elevated with prolonged bed rest or immobilization [82]. In fact, SOST knockout mice are protect from disuse-induced bone loss [83]. Conversely, overexpression of sclerostin in osteocytes prevents load-induced bone formation [24]. All together, these findings confirm that osteocytes, through the modulation of sclerostin and RANKL production, are responsible of the bone loss that follows reduced loading or disuse.

\section{Osteocytes and Cancer-Induced Bone Disease}

The bone is a frequent and preferred site for cancer metastases and cancer involvement. At least $80 \%$ of patients with advanced breast cancer and prostate cancer develop bone metastases, and $>90 \%$ of multiple myeloma (MM) patients experience bone involvement. Cancer of the bone is an important contributor to the decreased survival and quality of life for these patients due to bone pain and pathologic fractures. Tumor alters normal bone remodeling, resulting in the uncoupled activity of osteoclast and osteoblasts [84]. Enhanced osteoclast activity, in turn, causes osteolytic bone lesions and increases the growth and progression of the tumor in the bone. The bone marrow microenvironment in cancers that involve the bone is a major contributor to tumor growth and the bone destructive process. Numerous pieces of evidence demonstrate that both direct cell-to-cell interactions and exchange of soluble factors between tumor cells and cells in the bone marrow (osteoblasts, osteoclasts, stromal cells, and immune cells) stimulate osteoclast differentiation and alter osteoblast numbers and activity [84-86]. However, although our knowledge of osteocyte biology has increased during the last decade, the potential interactions between tumors and osteocytes and their contribution to the development and progression of cancer involving bone are just starting to be revealed. Accumulating evidence supports the notion that osteocytes play an important role in MM disease. MM is a hematologic malignancy characterized by monoclonal plasma cells that grow in the bone marrow and the presence of localized osteolytic lesions that rarely heal due to increased bone resorption and suppressed bone formation [87]. Previous work demonstrates that the BRC is disrupted in MM [88], suggesting that not only exchange of soluble factors occurs between $\mathrm{MM}$ and other cells in bone, but also mechanisms involving direct cell-tocell contact exist between different cells in the bone marrow microenvironment. In fact, we found that osteocytes physically interact (via Notch signaling) and exchange soluble factors with MM tumor cells in vivo [89•]. In addition, consistent with a recent study reporting decreased viability of osteocytes in biopsies from MM patients [90], our findings indicate that activation of Notch signaling in osteocytes by cell-to-cell contact with MM cells and MM-secreted factors increase the prevalence of apoptotic osteocytes in tumor-bearing bones (Delgado-Calle, Bellido, and Roodman, unpublished). The increased osteocyte apoptosis induced by MM cells enhanced the osteoclastogenic activity of osteocytes by increasing the ability of osteocytes to attract osteoclast precursors and their RANKL production, suggesting that apoptotic osteocytes in MM can start and/or sustain targeted bone resorption within the focal lesions observed in MM patients.

Further, the levels of the osteocyte specific product sclerostin are elevated in the serum of MM patients, and these levels correlate with reduced osteoblast function and poor survival [91], although the source of sclerostin remains unknown. We found in our studies that interactions between MM cells and osteocytes increased osteocytic sclerostin [92], which in turn decreases Wnt signaling and inhibits osteoblast differentiation, supporting the idea that osteocytes are the main source of sclerostin in MM. Importantly, our data also indicates that bidirectional Notch signaling between osteocytes and MM cells also stimulates MM cell growth. 
In addition to these results, recent findings suggest that FGF23 derived from osteocytes drives MM growth in bone by binding to FGF23 receptors and the coreceptor Klotho in MM cells and activating transcription of pro-metastatic and pro-osteolytic genes, such as heparanase [93]. Furthermore, osteocytes located in osteolytic lesions in MM patients overproduce interleukin-11 (IL-11), which is sufficient to enhance osteoclast differentiation [90]. New evidence also suggests that tumor growth induces changes in physical forces that are sensed by osteocytes, which enhance the production of factors such as chemokine (C-C motif) ligand 5 (CCL5) and matrix metalloproteinases that promote tumor progression and invasion [94]. Together, these results reveal a previously unknown role for osteocytes in cancer in bone and indicate that tumor cells instruct osteocytes to generate a microenvironment that favors tumor growth and bone destruction.

\section{Osteocytes and Therapies of Common Skeletal Disorders}

Evidence accumulated in the last decades supports the notion that current therapies for osteoporosis act on osteocytes (Fig. 2). The most widely prescribed and first-line drugs for common bone diseases include PTH and bisphosphonates such as alendronate, risedronate, ibandronate, or zoledronic acid. PTH is classically considered to be a bone catabolic agent; however, when delivered intermittently at low doses, PTH strongly stimulates cortical and/or trabecular bone formation in animal and clinical studies [95]. The mechanisms underlying PTH actions in the skeleton and the target cells for PTH in bone remain under intensive investigation. Most likely, PTH affects different signaling pathways and alters the activity of several bone cells, including osteoblasts, lining cells, osteoclasts, and osteocytes. Nonetheless, some skeletal actions of PTH might be mediated by direct effects of the hormone on osteocytes. Indeed, earlier work from our laboratory shows that whereas intermittent PTH administration increases bone mass and the circulating markers of bone resorption in an animal model, genetic knockdown of the PTH receptor 1 (PTHR1) in osteocytes blunts this effect, indicating that PTH receptor in osteocytes is required for bone anabolism induced by intermittent PTH administration [96, 97•]. Moreover, constitutive activation of PTHR1 signaling in osteocytes is sufficient to increase bone mass and the rate of bone remodeling, two recognized skeletal actions of PTH [50].

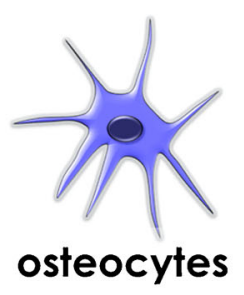

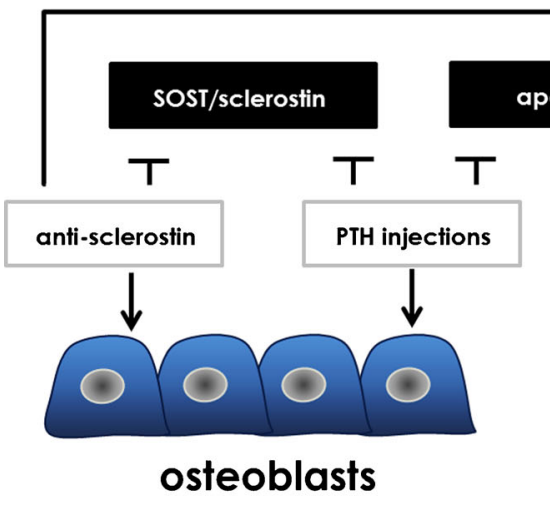

bone formation

Fig. 2 Osteocytes and therapies of common skeletal disorders. All current therapies for osteoporosis act on osteocytes. Anti-sclerostin antibodies increase bone formation and might reduce resorption by increasing OPG expression. The anabolic effect of intermittent PTH might be explained in part by downregulation of the SOST gene and therefore decreasing sclerostin levels. Part of the anti-fracture efficacy

(1) 
Whereas increased bone mass induced by PTH could be potentially explained by downregulation of the osteocyte-specific bone formation inhibitor SOST [11, 12, 20-22], the mechanism by which osteocytes mediate bone resorption induced by PTH remains unclear. The constitutive activation of PTHR1 signaling in osteocytes enhances bone resorption by regulating the expression of the RANKL gene directly in osteocytes through the distal control region (DCR) of the RANKL gene as it was discussed above (Section "Regulation of Bone Resorption by Osteocytes") [44]. Further, recent findings from our lab suggest that matrix metalloproteinase 14 (MMP14) is also a target gene of the osteocytic PTH receptor involved in the $\mathrm{PTH}$-mediated regulation of bone resorption (Delgado-Calle and Bellido, unpublished). MMP14 has been shown to be required for bone formation and maintenance of osteocytic processes during skeletal development $[98,99]$ and is also involved in RANKL shedding [100]. However, its potential role in PTH regulation of bone remodeling is unknown. In our studies, inhibition of MMP14 activity by a neutralizing antibody in animals with constitutive activation of PTHR1 signaling in osteocytes decreases bone resorption and the number of osteoclasts, and this effect is associated with decreased bone formation and mineral apposition.

Besides PTH analogs, all therapies for osteoporosis are anti-resorptive, including the previously mentioned bisphosphonates, the selective estrogen receptor modulators, estrogen preparations, and denosumab. In fact, the antifracture effects of treatment with sex steroids or bisphosphonates are partially explained by preservation of osteocyte viability [71, 101]. Similarly, inhibition of osteoblast apoptosis by intermittent administration of PTH also increases osteocyte density [102]. In addition, the potent anti-resorptive effects of the neutralizing antibodies for RANKL exert in bone might be due to the inhibition of osteocytic RANKL [103].

On the other hand, the discovery of osteocyte-mediated inhibition of Wnt signaling by sclerostin production has led to a novel anabolic approach for the management of osteoporosis: neutralization of sclerostin. Functional mutations of the SOST gene cause abnormal skeletal phenotypes in humans characterized by high bone mineral density [104, 105]. Genetic ablation of the SOST gene in mice results in a high bone mass phenotype with increased BMD, bone volume, bone formation, and bone strength [18]. Inhibition of sclerostin action has a marked anabolic effect in several animal models, and it is currently in clinical trials for the treatment of postmenopausal osteoporosis. Initial and ongoing studies in humans to determine the effect of sclerostin inhibition on osteoporosis revealed promising increases in BMD and bone formation [106•]. However, whether this effect translates in anti-fracture efficacy is still unknown. Nevertheless, these results are encouraging and advocate for new studies to determine the effect of sclerostin inhibition in other skeletal diseases characterized by low bone density (chronic kidney disease or multiple myeloma). All these results together highlight the potential of targeting osteocytes and their derived factors to increase bone mass and strength in low bone mass disorders.

\section{Conclusions}

In closing, advances of the last few years provided experimental evidence demonstrating that osteocytes are critical regulators of bone homeostasis by controlling osteoblast and osteoclast activity. We now know that although osteoclasts and osteoblasts execute bone remodeling by resorbing and forming bone respectively, osteocytes, through changes in their life-span and the production of RANKL and sclerostin, tightly regulate physiological bone remodeling. Moreover, changes in osteocyte viability and production of osteocytederived molecules contribute to the pathogenesis of several skeletal diseases. The discovery of some of the molecular mechanisms by which osteocytes influence the function of osteoblasts and osteoclasts has already given rise to therapeutic approaches to alter osteocyte function such as neutralizaing antibodies for sclerostin. In addition, overwhelming evidence also shows that some of the positive effects that first-line treatments for bone diseases, like PTH, bisphosphonates, or sex steroids, exert their action on bone at least in part by acting on osteocytes. Further, osteocytes can also play a major role in the development and progression of cancer that grows in bone, providing the mechanistic basis for targeting osteocyte-tumor cell interactions as a novel treatment for cancer induced bone disease. It is expected that ongoing and future investigations will reveal the role of new molecules and mechanisms by which osteocytes, the most abundant and long-lived cells in bone, influence bone homeostasis, thereby increasing the number of potential targets of pharmacological intervention towards an improved management of bone diseases.

Acknowledgments This work was supported by the National Institutes of Health Grants (R01 DK076007, and S10-RR023710 to TB), the Veteran's Administration (Merit Review to TB), and the IBMS Gideon and Sevgi Rodan Fellowship (to JDC).

\section{Compliance with Ethics Guidelines}

Conflict of Interest Jesus Delgado-Calle and Teresita Bellido declare that they have no conflict of interest.

Human and Animal Rights and Informed Consent This article does not contain any studies with human or animal subjects performed by any of the authors. 


\section{References}

Papers of particular interest, published recently, have been highlighted as:

- Of importance

1. Parfitt AM. The cellular basis of bone turnover and bone loss: a rebuttal of the osteocytic resorption-bone flow theory. Clin Orthop. 1977;127:236-47.

2. Bonewald LF. The amazing osteocyte. J Bone Miner Res. 2011;26(2):229-38.

3. Bellido T. Osteocytes and their role in bone remodeling. Actual Osteol (Spanish). 2013;9(1):56-64.

4. Wang L, Ciani C, Doty SB, Fritton SP. Delineating bone's interstitial fluid pathway in vivo. Bone. 2004;34(3):499-509.

5. Fritton SP, Weinbaum S. Fluid and solute transport in bone: flowinduced mechanotransduction. Annu Rev Fluid Mech. 2009;41: 347-74.

6. Bonewald LF, Johnson ML. Osteocytes, mechanosensing and Wnt signaling. Bone. 2008;42(4):606-15.

7. Winkler DG, Sutherland MK, Geoghegan JC, Yu C, Hayes T, Skonier JE, et al. Osteocyte control of bone formation via sclerostin, a novel BMP antagonist. EMBO J. 2003;22(23): 6267-76.

8. Poole KE, Van Bezooijen RL, Loveridge N, Hamersma H, Papapoulos SE, Lowik CW, et al. Sclerostin is a delayed secreted product of osteocytes that inhibits bone formation. FASEB J. 2005;19(13):1842-4.

9. Van Bezooijen RL, Roelen BA, Visser A, Wee-Pals L, de Wilt E, Karperien M, et al. Sclerostin is an osteocyte-expressed negative regulator of bone formation, but not a classical BMP antagonist. J Exp Med. 2004;199(6):805-14.

10. Robling AG, Niziolek PJ, Baldridge LA, Condon KW, Allen MJ, Alam I, et al. Mechanical stimulation of bone in vivo reduces osteocyte expression of Sost/sclerostin. J Biol Chem. 2008;283(9):5866-75.

11. Keller H, Kneissel M. SOST is a target gene for PTH in bone. Bone. 2005;37(2):148-58.

12. Bellido T, Ali AA, Gubrij I, Plotkin LI, Fu Q, O'Brien CA, et al. Chronic elevation of PTH in mice reduces expression of sclerostin by osteocytes: a novel mechanism for hormonal control of osteoblastogenesis. Endocrinology. 2005;146(11):4577-83.

13. Bellido T. Osteocyte-driven bone remodeling. Calcif Tissue Int. 2013;94(1):25-34. This article provides a detailed review of the role of osteocytes in bone remodeling.

14. Paic F, Igwe JC, Nori R, Kronenberg MS, Franceschetti T, Harrington $\mathrm{P}$, et al. Identification of differentially expressed genes between osteoblasts and osteocytes. Bone. 2009;45(4):682-92.

15. Choi HY, Dieckmann M, Herz J, Niemeier A. Lrp4, a novel receptor for dickkopf 1 and sclerostin, is expressed by osteoblasts and regulates bone growth and turnover in vivo. PLoS One. 2009;4(11), e7930.

16. Baron R, Kneissel M. WNT signaling in bone homeostasis and disease: from human mutations to treatments. Nat Med. 2013;19(2):179-92. This paper reviews the mechanisms by which WNT signaling regulates bone homeostasis.

17. Li J, Sarosi I, Cattley RC, Pretorius J, Asuncion F, Grisanti M, et al. Dkk1-mediated inhibition of Wnt signaling in bone results in osteopenia. Bone. 2006;39(4):754-66.

18. Li X, Ominsky MS, Niu QT, Sun N, Daugherty B, D'Agostin D, et al. Targeted deletion of the sclerostin gene in mice results in increased bone formation and bone strength. J Bone Miner Res. 2008;23(6):860-9.
19. Chang MK, Kramer I, Huber T, Kinzel B, Guth-Gundel S, Leupin $\mathrm{O}$, et al. Disruption of Lrp4 function by genetic deletion or pharmacological blockade increases bone mass and serum sclerostin levels. Proc Natl Acad Sci U S A. 2014;111(48):E5187-95. Experiments performed in this article report the role of LRP4 in bone homeostasis by retaining and facilitating sclerostin action locally.

20. van Lierop AH, Witteveen J, Hamdy N, Papapoulos S. Patients with primary hyperparathyroidism have lower circulating sclerostin levels than euparathyroid controls. Eur J Endocrinol. 2010;163(5):833-7.

21. Drake MT, Srinivasan B, Modder UI, Peterson JM, McCready LK, Riggs BL, et al. Effects of parathyroid hormone treatment on circulating sclerostin levels in postmenopausal women. J Clin Endocrinol Metab. 2010;95(11):5056-62.

22. Mirza FS, Padhi ID, Raisz LG, Lorenzo JA. Serum sclerostin levels negatively correlate with parathyroid hormone levels and free estrogen index in postmenopausal women. J Clin Endocrinol Metab. 2010;95(4):1991-7.

23. Robling AG, Turner $\mathrm{CH}$. Mechanical signaling for bone modeling and remodeling. Crit Rev Eukaryot Gene Expr. 2009;19(4):31938.

24. Tu X, Rhee Y, Lee R, Benson JD, Condon KW, Bivi N, et al. Downregulation of Sost/sclerostin expression is required for the osteogenic response to mechanical loading. J Bone Miner Res. 2010;25(Supp11):S87.

25. You L, Temiyasathit S, Lee P, Kim CH, Tummala P, Yao W, et al. Osteocytes as mechanosensors in the inhibition of bone resorption due to mechanical loading. Bone. 2008;42(1):172-9.

26. Tatsumi S, Ishii K, Amizuka N, Li M, Kobayashi T, Kohno K, et al. Targeted ablation of osteocytes induces osteoporosis with defective mechanotransduction. Cell Metab. 2007;5(6):464-75.

27. Fujita K, Xing Q, Khosla S, Monroe DG. Mutual enhancement of differentiation of osteoblasts and osteocytes occurs through direct cell-cell contact. J Cell Biochem. 2014;115(11):2039-44.

28. Zanotti S, Canalis E. Notch signaling in skeletal health and disease. Eur J Endocrinol. 2013;168(6):R95-103.

29. Canalis E, Parker K, Feng JQ, Zanotti S. Osteoblast lineagespecific effects of notch activation in the skeleton. Endocrinology. 2013;154(2):623-34.

30. Canalis E, Parker K, Feng J, Zanotti S. Expression of Notch in osteocytes prevents disuse osteoporosis. J Bone Miner Res. 2012;27(Supp11):S14.

31. Zanotti S, Canalis E. Notch regulation of bone development and remodeling and related skeletal disorders. Calcif Tissue Int. 2012;90(2):69-75.

32. Tu X, Delgado-Calle J, Condon KW, Maycas M, Zhang H, Carlesso N, et al. Osteocytes mediate the anabolic actions of canonica Wnt $/ \beta$-catenin signaling in bone. Proc Natl Acad Sci U S A. 2015;112(5):E478-86. This manuscript reports that activation of $\beta$-catenin signaling in osteocytes increases both osteoclasts and osteoblasts, leading to bone gain, and $\mathrm{d}$ identify osteocytes as central target cells of the anabolic actions of canonical Wnt/ $\beta$-catenin signaling in bone.

33. Glass DA, Karsenty G. In vivo analysis of Wnt signaling in bone. Endocrinology. 2007;148(6):2630-4.

34. White KE, Larsson TE, Econs MJ. The roles of specific genes implicated as circulating factors involved in normal and disordered phosphate homeostasis: frizzled related protein-4, matrix extracellular phosphoglycoprotein, and fibroblast growth factor 23. Endocr Rev. 2006;27(3):221-41.

35. Shimada T, Urakawa I, Yamazaki Y, Hasegawa H, Hino R, Yoneya T, et al. FGF-23 transgenic mice demonstrate hypophosphatemic rickets with reduced expression of sodium phosphate cotransporter type IIa. Biochem Biophys Res Commun. 2004;314(2):409-14. 
36. Rhee Y, Bivi N, Farrow EG, Lezcano V, Plotkin LI, White KE, et al. Parathyroid hormone receptor signaling in osteocytes increases the expression of fibroblast growth factor- 23 in vitro and in vivo. Bone. 2011;49(4):636-43.

37. Aguirre JI, Plotkin LI, Stewart SA, Weinstein RS, Parfitt AM, Manolagas SC, et al. Osteocyte apoptosis is induced by weightlessness in mice and precedes osteoclast recruitment and bone loss. J Bone Miner Res. 2006;21(4):605-15.

38. Almeida M, Han L, Martin-Millan M, Plotkin LI, Stewart SA, Roberson PK, et al. Skeletal involution by age-associated oxidative stress and its acceleration by loss of sex steroids. J Biol Chem. 2007;282(37):27285-97.

39. Schaffler MB, Cheung WY, Majeska R, Kennedy O. Osteocytes: Master Orchestrators of Bone. Calcif Tissue Int. 2013;94(1):5-24.

40. Noble BS, Stevens H, Loveridge N, Reeve J. Identification of apoptotic changes in osteocytes in normal and pathological human bone. Bone. 1997;20(3):273-82.

41. Yang J, Shah R, Robling AG, Templeton E, Yang H, Tracey KJ, et al. HMGB1 is a bone-active cytokine. J Cell Physiol. 2008;214(3):730-9.

42. Jilka RL, Noble B, Weinstein RS. Osteocyte apoptosis. Bone. 2012;54(2):264-71.

43. O'Brien CA, Nakashima T, Takayanagi H. Osteocyte control of osteoclastogenesis. Bone. 2013;54(2):258-63. This manuscript summarizes the mechanisms whereby osteocytes control osteoclast formation and bone resorption.

44. Ben-Awadh A, Delgado-Calle J, Tu X, Kuhlenschmidt K, Allen MR, Plotkin LI, et al. Parathyroid hormone receptor signaling induces bone resorption in the adult skeleton by directly regulating the RANKL gene in osteocytes. Endocrinology. 2014;155(8): 2797-809.

45. Hikita A, Yana I, Wakeyama H, Nakamura M, Kadono Y, Oshima $\mathrm{Y}$, et al. Negative regulation of osteoclastogenesis by ectodomain shedding of receptor activator of NF-kappaB ligand. J Biol Chem. 2006;281(48):36846-55

46. Honma M, Ikebuchi Y, Kariya Y, Hayashi M, Hayashi N, Aoki S, et al. RANKL subcellular trafficking and regulatory mechanisms in osteocytes. J Bone Miner Res. 2013;28(9):1963-49.

47. Boyce BF, Xing L. Functions of RANKL/RANK/OPG in bone modeling and remodeling. Arch Biochem Biophys. 2008;473(2): $139-46$.

48. Kramer I, Halleux C, Keller H, Pegurri M, Gooi JH, Weber PB, et al. Osteocyte Wnt/beta-catenin signaling is required for normal bone homeostasis. Mol Cell Biol. 2010;30(12):3071-85.

49. Glass DA, Bialek P, Ahn JD, Starbuck M, Patel MS, Clevers H, et al. Canonical Wnt signaling in differentiated osteoblasts controls osteoclast differentiation. Dev Cell. 2005;8(5):751-64.

50. O'Brien CA, Plotkin LI, Galli C, Goellner J, Gortazar AR, Allen $\mathrm{MR}$, et al. Control of bone mass and remodeling by PTH receptor signaling in osteocytes. PLoS One. 2008;3(8), e2942.

51. Islam S, Hassan F, Tumurkhuu G, Dagvadorj J, Koide N, Naiki Y, et al. Receptor activator of nuclear factor-kappa B ligand induces osteoclast formation in RAW 264.7 macrophage cells via augmented production of macrophage-colony-stimulating factor. Microbiol Immunol. 2008;52(12):585-90.

52. Bellido T, Plotkin LI, Bruzzaniti A. Bone cells. In: Burr D, Allen M, editors. Basic and Applied Bone Biology. 1st ed. Atlanta: Elsevier; 2014. p. 27-45

53. Zhao S, Zhang YK, Harris S, Ahuja SS, Bonewald LF. MLO-Y4 osteocyte-like cells support osteoclast formation and activation. J Bone Miner Res. 2002;17(11):2068-79.

54. Harris SE, MacDougall M, Horn D, Woodruff K, Zimmer SN, Rebel VI, et al. Meox2Cre-mediated disruption of CSF-1 leads to osteopetrosis and osteocyte defects. Bone. 2012;50(1):42-53.

55. Qing H, Ardeshirpour L, Pajevic PD, Dusevich V, Jahn K, Kato $\mathrm{S}$, et al. Demonstration of osteocytic perilacunar/ canalicular remodeling in mice during lactation. J Bone Miner Res. 2012;27(5):1018-29.

56. Tang SY, Herber RP, Ho SP, Alliston T. Matrix metalloproteinase13 is required for osteocytic perilacunar remodeling and maintains bone fracture resistance. J Bone Miner Res. 2012;27(9):1936-50.

57. Atkins GJ, Findlay DM. Osteocyte regulation of bone mineral: a little give and take. Osteoporos Int. 2012;23(8):2067-79.

58. Clarke MV, Russell PK, Findlay DM, Sastra S, Anderson PH, Skinner JP, et al. A role for the calcitonin receptor to limit bone loss during lactation in female mice by inhibiting osteocytic osteolysis. Endocrinology. 2015;156(9):3203-14.

59. Belanger LF, Belanger C, Semba T. Technical approaches leading to the concept of osteocytic osteolysis. Clin Orthop Relat Res. 1967;54:187-96.

60. Hu H, Hilton MJ, Tu X, Yu K, Ornitz DM, Long F. Sequential roles of Hedgehog and Wnt signaling in osteoblast development. Development. 2005;132(1):49-60.

61. Day TF, Guo X, Garrett-Beal L, Yang Y. Wnt/beta-catenin signaling in mesenchymal progenitors controls osteoblast and chondrocyte differentiation during vertebrate skeletogenesis. Dev Cell. 2005;8(5):739-50.

62. Hill TP, Spater D, Taketo MM, Birchmeier W, Hartmann C. Canonical Wnt/beta-catenin signaling prevents osteoblasts from differentiating into chondrocytes. Dev Cell. 2005;8(5):727-38.

63. Rodda SJ, McMahon AP. Distinct roles for Hedgehog and canonical Wnt signaling in specification, differentiation and maintenance of osteoblast progenitors. Development. 2006;133(16): 3231-44.

64. Holmen SL, Zylstra CR, Mukherjee A, Sigler RE, Faugere MC, Bouxsein ML, et al. Essential role of beta-catenin in postnatal bone acquisition. J Biol Chem. 2005;280(22):21162-8.

65. Wijenayaka AR, Kogawa M, Lim HP, Bonewald LF, Findlay DM, Atkins GJ. Sclerostin stimulates osteocyte support of osteoclast activity by a RANKL-dependent pathway. PLoS One. 2011;6(10), e25900.

66. Stolina M, Dwyer D, Niu QT, Villasenor KS, Kurimoto P, Grisanti $\mathrm{M}$, et al. Temporal changes in systemic and local expression of bone turnover markers during six months of sclerostin antibody administration to ovariectomized rats. Bone. 2014;67:305-13.

67. Manolagas SC, Parfitt AM. What old means to bone. Trends Endocrinol Metab. 2010;21(6):369-74.

68. Delgado-Calle J, Arozamena J, Garcia-Renedo R, Garcia-Ibarbia C, Pascual-Carra MA, Gonzalez-Macias J, et al. Osteocyte Deficiency in Hip Fractures. Calcif Tissue Int. 2011;89(4):32734.

69. Manolagas SC. From Estrogen-Centric to Aging and Oxidative Stress: A Revised Perspective of the Pathogenesis of Osteoporosis. Endocr Rev. 2010;31:266-300.

70. Weinstein RS, Wan C, Liu Q, Wang Y, Almeida M, O'Brien CA, et al. Endogenous glucocorticoids decrease skeletal angiogenesis, vascularity, hydration, and strength in 21-month-old mice. Aging Cell. 2009;9(2):147-61.

71. Kousteni S, Bellido T, Plotkin LI, O'Brien CA, Bodenner DL, Han $\mathrm{L}$, et al. Nongenotropic, sex-nonspecific signaling through the estrogen or androgen receptors: dissociation from transcriptional activity. Cell. 2001;104(5):719-30.

72. Boyce BF, Xing L, Jilka RL, Bellido T, Weinstein RS, Parfitt AM, et al. Apoptosis in bone cells. In: Bilezikian JP, Raisz LG, Rodan GA, editors. Principles of Bone Biology. 2nd ed. San Diego: Academic Press; 2002. p. 151-68.

73. Weinstein RS. Clinical practice. Glucocorticoid-induced bone disease. N Engl J Med. 2011;365(1):62-70.

74. Modder UI, Hoey KA, Amin S, McCready LK, Achenbach SJ, Riggs BL, et al. Relation of age, gender, and bone mass to circulating sclerostin levels in women and men. J Bone Miner Res. 2010;26(2):373-9. 
75. Noble BS, Peet N, Stevens HY, Brabbs A, Mosley JR, Reilly GC, et al. Mechanical loading: biphasic osteocyte survival and the targeting of osteoclasts for bone destruction in rat cortical bone. Am J Physiol Cell Physiol. 2003;284(4):C934-43.

76. Cardoso L, Herman BC, Verborgt O, Laudier D, Majeska RJ, Schaffler MB. Osteocyte apoptosis controls activation of intracortical resorption in response to bone fatigue. J Bone Miner Res. 2009;24(4):597-605.

77. Verborgt O, Tatton NA, Majeska RJ, Schaffler MB. Spatial distribution of Bax and Bcl-2 in osteocytes after bone fatigue: complementary roles in bone remodeling regulation? J Bone Miner Res. 2002;17(5):907-14.

78. Verborgt O, Gibson G, Schaffler MB. Loss of osteocyte integrity in association with microdamage and bone remodeling after fatigue in vivo. J Bone Miner Res. 2000;15(1):60-7.

79. Xiong J, Onal M, Jilka RL, Weinstein RS, Manolagas SC, O'Brien CA. Matrix-embedded cells control osteoclast formation. Nat Med. 2011;17(10):1235-41.

80. Plotkin LI, de Gortazar AR, Davis HM, Condon KW, Gabilondo $\mathrm{H}$, Maycas M, et al. Inhibition of Osteocyte Apoptosis Prevents the Increase in Osteocytic RANKL but it does not Stop Bone Resorption or the Loss of Bone Induced by Unloading. J Biol Chem. 2015;290(31):18934-42. Experiments performed in this manuscript demonstrate a functional relationship between osteocyte apoptosis and RANKL production.

81. Kennedy OD, Laudier DM, Majeska RJ, Sun HB, Schaffler MB. Osteocyte apoptosis is required for production of osteoclastogenic signals following bone fatigue in vivo. Bone. 2014;64:132-7. Experiments performed in this manuscript demonstrate a functional relationship between osteocyte apoptosis and RANKL production.

82. Gaudio A, Pennisi P, Bratengeier C, Torrisi V, Lindner B, Mangiafico RA, et al. Increased sclerostin serum levels associated with bone formation and resorption markers in patients with immobilization-induced bone loss. J Clin Endocrinol Metab. 2010;95(5):2248-53

83. Lin C, Jiang X, Dai Z, Guo X, Weng T, Wang J, et al. Sclerostin mediates bone response to mechanical unloading through antagonizing Wnt/beta-catenin signaling. J Bone Miner Res. 2009;24(10):1651-61.

84. Weilbaecher KN, Guise TA, McCauley LK. Cancer to bone: a fatal attraction. Nat Rev Cancer. 2011;11(6):411-25.

85. Yoneda T, Hiraga T. Crosstalk between cancer cells and bone microenvironment in bone metastasis. Biochem Biophys Res Commun. 2005;328(3):679-87.

86. Roodman GD. Role of the bone marrow microenvironment in multiple myeloma. J Bone Miner Res. 2002;17(11):1921-5.

87. Roodman GD. Pathogenesis of myeloma bone disease. J Cell Biochem. 2010;109(2):283-91.

88. Andersen TL, Soe K, Sondergaard TE, Plesner T, Delaisse JM. Myeloma cell-induced disruption of bone remodelling compartments leads to osteolytic lesions and generation of osteoclastmyeloma hybrid cells. Br J Haematol. 2010;148(4):551-61.

89. Delgado-Calle J, Bellido T, Roodman GD. Role of osteocytes in multiple myeloma bone disease. Curr Opin Support Palliat Care. 2014;8(4):407-13. This article reviews the contribution of osteocytes to multiple myeloma disease.

90. Giuliani N, Ferretti M, Bolzoni M, Storti P, Lazzaretti M, Dalla $\mathrm{PB}$, et al. Increased osteocyte death in multiple myeloma patients: role in myeloma-induced osteoclast formation. Leukemia. 2012;26(6):1391-401

91. Terpos E, Christoulas D, Katodritou E, Bratengeier C, Gkotzamanidou M, Michalis E, et al. Elevated circulating sclerostin correlates with advanced disease features and abnormal bone remodeling in symptomatic myeloma: reduction postbortezomib monotherapy. Int J Cancer. 2012;131(6):1466-71.

92. Delgado-Calle J, Bellido T, Roodman GD. Direct Cell-To-Cell Interactions Between Osteocytes and Multiple Myeloma (MM) Cells Upregulate Sost and Downregulate OPG Expression In Osteocytes: Evidence For Osteocytic Contributions To MMInduced Bone Disease. Blood. 2013;122(21):3140.

93. Suvannasankha A, Tompkins DR, Edwards DF, Petyaykina KV, Crean CD, Fournier PG, et al. FGF23 is elevated in multiple myeloma and increases heparanase expression by tumor cells. Oncotarget. 2015;6(23):19647-60.

94. Sottnik JL, Dai J, Zhang H, Campbell B, Keller ET. Tumorinduced pressure in the bone microenvironment causes osteocytes to promote the growth of prostate cancer bone metastases. Cancer Res. 2015;75(11):2151-8.

95. Silva BC, Costa AG, Cusano NE, Kousteni S, Bilezikian JP. Catabolic and Anabolic Actions of Parathyroid Hormone on the Skeleton. J Endocrinol Investig. 2011;34(10):801-10.

96. Tu X, McAndrews K, Delgado-Calle J, Olivos N, Ben-Awadh A, Kim W, et al. Osteocytic PTH receptor is required for bone anabolism induced by intermittent PTH administration, but is dispensable for bone resorption and the loss of bone induced by chronic PTH elevation. J Bone Miner Res. 2013;28(Suppl1):S233.

97. Bellido T, Saini V, Divieti Pajevic P. Effects of PTH on osteocyte function. Bone. 2013;54:250-7. This review summarizes the mechanisms by which osteocytes mediate the actions of PTH on bone.

98. Sato T, del Carmen OM, Hou P, Heegaard AM, Kumegawa M, Foged NT, et al. Identification of the membrane-type matrix metalloproteinase MT1-MMP in osteoclasts. J Cell Sci. 1997;110(Pt 5):589-96.

99. Holmbeck K, Bianco P, Caterina J, Yamada S, Kromer M, Kuznetsov SA, et al. MT1-MMP-deficient mice develop dwarfism, osteopenia, arthritis, and connective tissue disease due to inadequate collagen turnover. Cell. 1999;99(1):81-92.

100. Sabbota AL, Kim HR, Zhe X, Fridman R, Bonfil RD, Cher ML. Shedding of RANKL by tumor-associated MT1-MMP activates Src-dependent prostate cancer cell migration. Cancer Res. 2010;70(13):5558-66.

101. Bellido T, Plotkin LI. Novel actions of bisphosphonates in bone: Preservation of osteoblast and osteocyte viability. Bone. 2011;49: $50-5$.

102. Jilka RL, Weinstein RS, Bellido T, Roberson P, Parfitt AM, Manolagas SC. Increased bone formation by prevention of osteoblast apoptosis with parathyroid hormone. J Clin Invest. 1999;104(4):439-46.

103. McClung MR, Lewiecki EM, Cohen SB, Bolognese MA, Woodson GC, Moffett AH, et al. Denosumab in postmenopausal women with low bone mineral density. N Engl J Med. 2006;354(8):821-31.

104. Balemans W, Ebeling M, Patel N, Van Hul E, Olson P, Dioszegi $\mathrm{M}$, et al. Increased bone density in sclerosteosis is due to the deficiency of a novel secreted protein (SOST). Hum Mol Genet. 2001;10(5):537-43.

105. Balemans W, Patel N, Ebeling M, Van Hul E, Wuyts W, Lacza C, et al. Identification of a $52 \mathrm{~kb}$ deletion downstream of the SOST gene in patients with van Buchem disease. J Med Genet. 2002;39(2):91-7.

106. Shah AD, Shoback D, Lewiecki EM. Sclerostin inhibition: a novel therapeutic approach in the treatment of osteoporosis. Int $\mathrm{J}$ Womens Health. 2015;7:565-80. In this review, the authors discuss the biology of Wnt signaling and sclerostin inhibition, and the consequences of sclerostin inhibition in animal models and clinical trials. 